\title{
ONCOCHART Management Tool
}

National Cancer Institute

\section{Source}

National Cancer Institute. ONCOCHART Management Tool. NCI Thesaurus. Code

C105669.

A proprietary charge capture, documentation and electronic medical record system for radiation oncology. 